Selbstregelung über freie Träger, Wohnungsunternehmen oder selbstständige Wohnberater*innen (vgl. Kapitel 4.2.1). Vielfach sind es zudem Kommunen, die im Rahmen ihres kommunalen Aufgabenspektrums Wohnberatungen anbieten und dabei - teilweise - durch landespolitische koordinierende Strukturen unterstützt werden. Joo (2018) hat diese Gemengelage unter dem Schlagwort »Wohlfahrtskorporatismus« untersucht. In ihrer Lesart findet eine "Sozialpolitisierung der vormals privaten Räume« statt, wobei der Markt der Wohnberatung aktuell durch ein »komplexes Verhältnis zwischen marktwirtschaftlichen und sozialpolitischen Elementen gekennzeichnet « ist (Joo 2018: 162ff.). Zwar ist mit der Sozialpolitisierung eine zunehmende politische Steuerung verbunden, jedoch kann damit noch keine Aussage darüber getroffen werden, wie qualitativ hochwertig die tatsächlichen Beratungen letztlich sind. Je nach Struktur vor Ort seien gerade die kurzen Wege wichtig und mehrere Wohnberater*innen bewerten diese von außen verworrene Struktur unterschiedlichster Beratungsakteure nicht unbedingt als negativ. So könne z.B. eine Integration in bereits bestehende Beratungsangebote sinnvoller sein als der Aufbau einer Parallelstruktur (Wohnberatungsstelle-3: 25). Aus politikfeldanalytischer Perspektive erschweren diese politikfeldübergreifenden Beratungsangebote die Analyse allerdings. Insbesondere die Pflegekassen sind ein Akteur, der aus wohnungspolitischer Sicht bislang noch nicht aufgeschienen war. Hier zeigt sich klar der Querschnittscharakter des barrierefreien bzw. -reduzierten Wohnraums, der einerseits in wohnungspolitischen und andererseits in sozialpolitischen Handlungsfeldern zu verorten ist.

\title{
6.4 Fazit
}

Kapitel 6 hat untersucht, in welchen Ausprägungen die Bundesländer politische Maßnahmen zur Steuerung von barrierefreiem bzw. -reduziertem Wohnraum nutzen. Diese Erkenntnisse werden im Folgenden zusammengefasst. Zur Übersicht dient zunächst der zugrundeliegende Policy-Mix, der auf Basis der gewonnenen Erkenntnisse eine inhaltliche Verfeinerung erfährt. Daraufhin sind die einzelnen Policy-Ausprägungen von Interesse: Welche Bundesländer konnten als besonders aktiv in der politischen Steuerung identifiziert werden? Diese Analyseergebnisse sind anschließend zu ergänzen und abzugleichen mit den Einschätzungen aus dem Feld - von den Interviewpartner*innen wurde dafür die Nennung sogenannter Vorreiter-Länder erbeten.

Policy-Mix zum barrierefreien bzw. -reduzierten Wohnraum

Tabelle 9 zeigt den untersuchten Policy-Mix zum regulativen Bauordnungsrecht, den Förderprogrammen der Landesförderbanken und den Wohnberatungsstellen. Dabei sind zwei Unterscheidungslinien wichtig, die im Rahmen der Analyse immer wieder deutlich geworden sind. 
Tabelle 9: Policy-Mix zum barrierefreien bzw. -reduzierten Wohnraum (eigene Darstellung)

\begin{tabular}{|c|c|c|}
\hline $\begin{array}{l}\text { Regulatives Instrumentari- } \\
\text { um: Bauordnungsrechtliche } \\
\text { Vorgaben zum barrierefreien } \\
\text { Bauen }\end{array}$ & $\begin{array}{l}\text { Ökonomisches Instrumenta- } \\
\text { rium: Förderprogramme zum } \\
\text { barrierefreien/-reduzierten } \\
\text { (Um-)Bau }\end{array}$ & $\begin{array}{l}\text { Informationelles Instrumen- } \\
\text { tarium: Wohnberatungen zur } \\
\text { Wohnungsanpassung }\end{array}$ \\
\hline \multicolumn{2}{|c|}{ Neubau } & \\
\hline & \multicolumn{2}{|c|}{ Bestandsumbau } \\
\hline \multicolumn{2}{|c|}{ Organisierte Wohnungs- und Bauwirtschaft } & \\
\hline & \multicolumn{2}{|c|}{ Privatvermieter*innen, Selbstnutzer*innen und Mieter*innen } \\
\hline
\end{tabular}

Die erste Unterscheidungslinie verläuft zwischen Neubau und Bestandsumbau, dargestellt in dunkelgrauen Schattierungen. So legt das Bauordnungsrecht barrierefreie Vorgaben für den Neubau fest - zu einem geringen Anteil auch für Umnutzungen im Bestand -, während Förderprogramme vielfach sowohl Neubauten als auch Bestandsumbauten finanziell unterstützen. Die Wohnberatungen indessen konzentrieren sich auf den individuellen Bestandsumbau und weisen durch die Finanzierungsberatung eine deutliche Schnittstelle zu den Förderprogrammen auf. In Neubauprojekte sind Wohnberater*innen eher selten involviert, wobei sich durch lokale Netzwerke durchaus Kooperationen mit Wohnungsunternehmen entwickeln können (vgl. Wohnberatungsstelle-6: 23). Tendenziell verläuft die Aufgabenteilung aber dahingehend, dass der barrierefreie Neubau zuvorderst über das Bauordnungsrecht und Förderprogramme politisch adressiert wird, wohingegen für die Barrierereduzierung im Bestand neben der finanziellen Förderung vor allem die Beratungsstellen zur Wohnungsanpassung zuständig sind.

Die zweite Unterscheidungslinie befasst sich mit den primären Steuerungsadressat*innen, dargestellt in hellgrauen Schattierungen. So adressiert das Bauordnungsrecht hauptsächlich die organisierte Wohnungs- und Bauwirtschaft. Da die Vorgaben zum barrierefreien Bauen in allen Bundesländern erst ab einer bestimmten Gebäudegröße gelten, werden Privatpersonen, die in Eigenregie ihr Eigenheim errichten, nicht von dieser Maßnahme erfasst. Förderungen wiederum bestehen sowohl für institutionelle wie auch für private Akteure des Wohnungsmarktes - auch wenn die einzelnen Bundesländer hier je nach den regionalen Wohnungsmärkten unterschiedliche Schwerpunkte setzen. Die Gruppe der privaten Kleinvermieter*innen, der Selbstnutzer*innen von Eigentum und der Mieter*innen wird neben der finanziellen Förderung insbesondere durch die Wohnberatungsstellen zur Wohnungsanpassung adressiert.

Beide Unterscheidungslinien korrelieren miteinander, verhelfen in ihrer Differenzierung aber zu einem besseren Verständnis des Untersuchungsgegenstandes und damit der politischen Steuerung zum barrierefreien bzw. -reduzierten Wohnraum.

\section{Die Policy-Ausprägungen in den 16 Bundesländern}

Die Typologie hatte das primäre Ziel, die Strukturen der bauordnungsrechtlichen Vorgaben zur Barrierefreiheit, der einschlägigen Förderprogramme sowie der Wohnberatungsstellen zur Wohnungsanpassung zu erarbeiten. Dabei war mit der vergleichenden 
Betrachtung von 16 Bundesländern auch verbunden, Cluster oder länderspezifische Besonderheiten zu identifizieren. Abbildung 25 fasst die gewonnen Erkenntnisse zusammen, indem sie die Länder darstellt, die in einzelnen Kategorien der drei untersuchten Policies als besonders aktiv hervorgeschienen sind.

\section{Abbildung 25: Empirische Erkenntnisse zur Typologie politischer Maßnahmen}
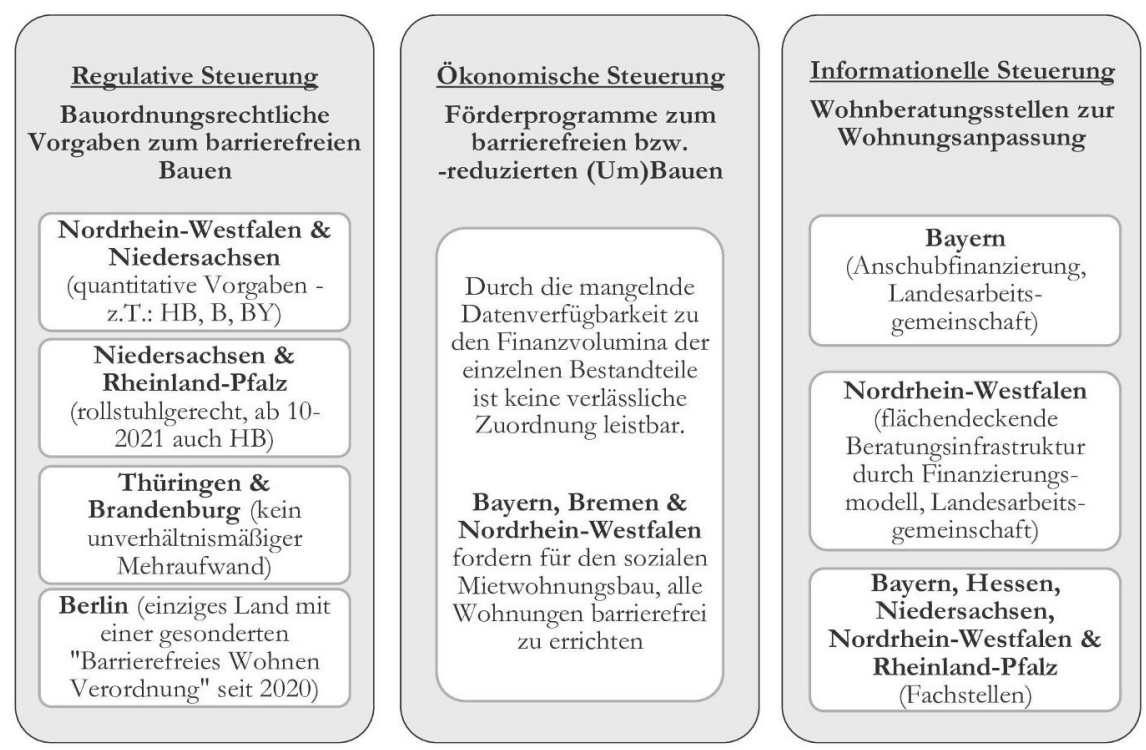

Diese Zusammenschau offenbart, dass die Bundesländer unterschiedliche Strategien zur politischen Steuerung von barrierefreiem bzw. -reduziertem Wohnraum verfolgen. Während Rheinland-Pfalz sich in seiner Landesbauordnung für eine verbindliche Quote zum rollstuhlgerechten Wohnraum entschieden hat, wählt Nordrhein-Westfalen den Weg, ab einer bestimmten Gebäudegröße alle Wohnungen barrierefrei, aber eingeschränkt mit dem Rollstuhl nutzbar errichten zu lassen. Niedersachsen kombiniert sogar verhältnismäßig strenge Vorgaben zum rollstuhlgerechten und zum eingeschränkt mit dem Rollstuhl nutzbaren Wohnraum. In der Wohnraumförderung ist eine Einschätzung einzelner Länder durch die Vielfalt an Förderprogrammen und die mangelnde Datenverfügbarkeit zu einzelnen Finanzvolumina nur eingeschränkt leistbar. Erkennbar ist aber, dass Bayern, Bremen und Nordrhein-Westfalen im sozialen Mietwohnungsbau alle Wohnungen verbindlich nach DIN 18040-2 festschreiben je mit den Ausnahmen, die in den Verwaltungsvorschriften Technische Baubestimmungen formuliert sind (StMB Bayern 2019: 4; MHKBG NRW 2020b: 50; BAB 2020: 4). Und auch bei der Wohnberatung zeigen sich Unterschiede von einer dauerhaften Kofinanzierung in Nordrhein-Westfalen über die Anschubfinanzierung in Bayern bis hin zu den weiter verbreiteten Fachstellen.

Insgesamt sind Niedersachen, Nordrhein-Westfalen sowie Rheinland-Pfalz die Bundesländer, die im untersuchten Policy-Mix als besonders aktiv bezeichnet werden können. Auch Bayern ist in diese Liste einzuordnen, da das Bauordnungsrecht sowie 
die Vorgaben in der sozialen Wohnraumförderung überdurchschnittlich »streng« sind und das Modell zur Unterstützung der Wohnberatungsstellen vielfach hervorgehoben wurde. Allerdings handelt es sich hier um eine dynamische Entwicklung. Bremen beispielsweise verschärft sein Bauordnungsrecht zum Jahr 2021 und sticht auch bislang durch seine soziale Wohnraumförderung und die Unterstützung der Wohnberatungsstelle komfort e.V. hervor. Und Berlin hat zum Januar 2020 als erstes Bundesland eine eigene Verordnung spezifisch zum Barrierefreien Bauen erlassen. Es zeigen sich also inkrementelle und in den einzelnen Bundesländern je unterschiedlich ablaufende Entwicklungen in der politischen Steuerung zum barrierefreien bzw. -reduzierten Wohnraum.

\section{Ergänzung der Analyseergebnisse durch Einschätzungen aus dem Feld}

Die bisherige Einschätzung auf Basis der Policy-Kriterien wird mit der Einschätzung der Interviewpartner*innen abgeglichen. Sowohl im Rahmen der vertiefenden Gespräche zu den Förderprogrammen und Wohnberatungsstellen, als auch in den Fallstudien wurde nach sogenannten Vorreiter-Ländern gefragt. Gemeint sind damit Länder, die diesen Regelungsbereich durch eine aktive politische Steuerung angehen und auf die auch aus anderen Bundesländern entsprechend verwiesen wird. Dabei zeigt sich, dass selbst einschlägige Expert*innen wie Sachverständige für barrierefreies Bauen oder die Zuständigen für soziale Wohnraumförderung in den Landesförderinstituten nur begrenzt aussagefähig waren, was die Policy-Strukturen in den übrigen Bundesländern betrifft.

Mit dieser Einschränkung vorab sind folgende Erkenntnisse aus dem Interviewmaterial zu gewinnen: Zum Bauordnungsrecht wird vielfach auf die Thüringer Bauordnung verwiesen, da diese ohne die umstrittene Klausel zum unverhältnismäßigen Mehraufwand auskommt (IP 1; IP 14; IP 26; IP 29). Zudem erfährt die nordrhein-westfälische Bauordnung bundesweite Aufmerksamkeit: Die von der früheren rot-grünen Landesregierung novellierte Fassung sollte Ende 2017 in Kraft treten, wurde jedoch von der schwarz-gelben Nachfolgeregierung für ein Jahr aufgeschoben. Das Moratorium wurde mit zusätzlichen Kostensteigerungen begründet, wobei Barrierefreiheit einen zentralen Streitpunkt darstellte (MHKBG NRW 2017, MHKBG NRW 2020a). Die Interviewpartner*innen beurteilen die daraufhin eingeführten Regelungen als ambivalent. Dass statt einer Quote zum rollstuhlgerechten Wohnraum nun Zielvereinbarungen mit Städten in Ballungsräumen geschlossen werden sollen, wird etwa von wohnungswirtschaftlicher Seite als positiv, von Betroffenenperspektive heraus indessen als kritisch gewertet (IP 12: 118; IP 14: 20; IP 23: 76; IP 29: 143). Weiterhin verweisen Interviewpartner*innen aus mehreren Bundesländern auf einen hessischen Nachweis zum barrierefreien Bauen (IP 29; IP 37; IP 56). Dieses »Barrierefrei-Konzept« ist in einem Bauvorlagenerlass geregelt. Es soll in der Planung und im Genehmigungsverfahren durch die Bauaufsichtsbehörden zu mehr Verbindlichkeit führen und kann als eine Art »Checkliste« eingesetzt werden (vgl. Kapitel 7.2.1).

Bezüglich der Förderprogramme zum barrierefreien bzw. -reduzierten Wohnraum konnten aus den Gesprächen mit den Landesförderinstituten keine validen Aussagen zu besonders aktiven Bundesländern abgeleitet werden. So trauen sich auch die Landes- 
förderbanken selbst keine entsprechende Beurteilung zu und verweisen auf die Kleinteiligkeit der einzelnen Förderprogramme und ihrer Bestandteile (IP 5; IP 17).

Die Strukturen zu den Wohnberatungsstellen zur Wohnungsanpassung konnten bereits durch die Analyse der Sekundärdaten und die Gespräche mit den regionalen Ansprechpartner*innen der Bundesarbeitsgemeinschaft gut verglichen werden. Neben den Vorreiter-Ländern Bayern und Nordrhein-Westfalen wurden die Fachstellen in Hessen (IP 1; IP 14) und Niedersachsen (IP 1; IP 13; IP 54) als positiv hervorgehoben. Das rheinland-pfälzische Modell mit der Anbindung an die Architektenkammer schien indessen weniger geläufig zu sein.

Abschließend sei darauf zu verweisen, dass die politische Steuerung in Bayern in das breitere Programm »Bayern barrierefrei« eingebettet ist: Im Jahr 2013 hat der damalige Ministerpräsident Horst Seehofer das Ziel formuliert, bis 2023 insbesondere den öffentlichen Raum barrierefrei zu gestalten. Aber auch die Wohnraumförderung ist in das Programm integriert und landesweit wurden 18 »Beratungsstellen Barrierefreiheit « eingerichtet (StMAS 2020). Ein derart umfassender Ansatz konnte in keinem anderen Bundesland nachgezeichnet werden und weist auf die Gedanken zur Politikintegration bzw. zum Disability Mainstreaming hin, nach denen Inklusion als Querschnittsthema in unterschiedlichste Politikfelder getragen wird (vgl. Kapitel 4.5.1).

\section{Fazit zur Typologie politischer Maßnahmen}

Zusammenfassend wird der barrierefreie bzw. -reduzierte Wohnraum durch einen Policy-Mix verschiedener politischer Maßnahmen gesteuert, die je eigene inhaltliche Schwerpunkte setzen. Das Bauordnungsrecht und die Förderprogramme sind in der Ressortbetrachtung vorwiegend der Wohnungspolitik zuzuordnen, während die Wohnberatung sozialpolitisch verankert ist. Hier weist Joo (2018: 80) auf die große Bedeutung des Gebäudebestandes hin, da insbesondere die Nutzergruppe der älteren Menschen vergleichsweise selten in Neubauten zöge. Eine rein wohnungspolitische und auf den Neubau begrenzte Analyse würde dem Untersuchungsgegenstand des barrierefreien bzw. -reduzierten Wohnraums also nur bedingt gerecht. Heinelt/Egner (2006: 203) erläutern dazu, dass der Verweis auf ein gesamtes Politikfeld ohnehin irreführen könne: "Sie [die einzelnen Maßnahmen, Anm. der Autorin]- und nicht per se ein Politikfeld als solches - werden von politischen Akteuren unterstützt oder hinterfragt und sind damit der eigentliche Bezugspunkt politischer Auseinandersetzung«. Die einzelnen Maßnahmen wiederum halten sich nicht immer an die strenge analytische Politikfeldlogik, womit politikfeldübergreifende Analysen unabdingbar werden.

Mit Rogge/Reichardt (2016: 1621ff.) und Cunningham et al. (2013: 4f.) wurde vorab herausgearbeitet, dass Policy-Mixes vielfach eher organisch wachsen, statt von den jeweiligen politischen Entscheider*innen strategisch geplant zu werden (vgl. Kapitel 4.2.1). Insbesondere bei der Beteiligung unterschiedlicher Ressorts erscheint dies naheliegend, wodurch isoliert voneinander verlaufende Policies mit ganz unterschiedlichen Logiken entstehen können. Allerdings sind beim barrierefreien bzw. -reduzierten Wohnraum durchaus Synergien zwischen den einzelnen Maßnahmen nachzuweisen, informieren doch die Beratungsstellen über Förderprogramme oder unterstützen die Landesförderbanken wiederum barrierefreie Kriterien, die über 
die bauordnungsrechtlichen Vorgaben hinausgehen. Durch diese Querverbindungen haben Änderungen in einer Policy Rückwirkungen auf die anderen Policies und können damit den gesamten Policy-Mix inkrementell verändern (vgl. auch Kivimaa/Kern 2016: 206). Dass politische Akteure dabei je nach ihrem Staatsverständnis unterschiedliche politische Instrumente präferieren und einige folglich stärker auf ordnungsrechtliche Vorgaben, andere stattdessen auf eine weichere ökonomische oder informationelle Steuerung setzen, wurde als »ideologischer Filter« herausgearbeitet (vgl. Kapitel 4.2.1). Dies wird in den folgenden Fallstudien zu Sachsen-Anhalt und Hessen genauer untersucht, wenn es darum geht, die zum beobachteten Policy-Mix führenden politischen Prozesse zu erklären. 